\title{
Arbuscular Mycorrhizal Fungi and Trichoderma harzianum Induced Resistance in Tomato Varieties Against Fusarium oxysporum f. sp. lycopersici
}

\author{
Karima G. H. ${ }^{1}$ and Hemmat A. Ibrahim ${ }^{2}$
}

${ }^{1}$ Plant Pathology Department, Faculty of Agriculture, Ain Shames University, Egypt.

${ }^{2}$ Agricultural Biochemistry Department, Faculty of Agriculture, Ain Shams University, Egypt. Received on: 6/2/2017

Accepted for publication on: 13/2/2017

\begin{abstract}
Pot experiments were conducted to study the effect of Arbuscular mycorrhizal fungi (AMF), Tricoderma harzianum and mix of them on suppression of fusarium wilt of three varieties of tomato (castle-rock, super marmande and peto 86). Disease severity, shoot and root Length, fresh, dry weights and the changes in amino acids, reducing sugars and phenolic compounds contents and phenylalanine ammonia lyase, ascorbate peroxidase, peroxidase and poly phenoloxidase activities were investigated.

The treatments AMF, T. harzianum and AMF + T. harzianum gave significant reduction of disease severity and elevated phenolic acids, amino acids and reducing sugars contents and POD, PPO, APX and PAL activities in roots and leaves of tomato varieties.
\end{abstract}

Keywords: Arbuscular mycorrhizal fungi, Tricoderma harzianum, tomato, Fusarium oxysporum, amino acids, reducing sugars, phenolic compounds, antioxidant enzymes.

\section{Introduction}

Tomatoes are one of the most widely cultivated vegetable crops in Egypt and in all countries either as open field or protected crops. Its fruit is rich in vitamins and is therefore used in salads, cooked as a vegetable or made into tomato paste and tomato sauce.

Tomato plants are affected by several diseases, including Fusarium wilt caused by Fusarium oxysporum f. sp. lycopersici (sacc.) Snyder and Hansen. This is a destructive disease of tomato worldwide (Jones, et al 1991). Methods used to control vascular wilt are either not very efficient or are difficult to apply. The best way to control the disease is by selecting resistant varieties of tomatoes. Although commercial varieties of tomato resistant to $F$. oxysporum f. sp. lycopersici races 1 and 2 are available, additional pathogenic strains and race 3 of the pathogen has been reported in several countries (Amini, 2009). For this reason, alternative methods of controlling the disease have to be explored inclusive of biological control methods. Adding biocontrol agents directly to the roots is an efficient and inexpensive means to provide a more vigorous transplant with disease protection when it is transplanted in the field (Nemec, et al 1996). Trichoderma species, that are common inhabitants of the rhizosphere are biological control organisms against a wide range of soil borne pathogens and also have been known to provide plant growth promotion. Trichoderma harzianum Rifai have been known as antagonistic to various root pathogens such as Pythium spp., Rhizoctonia spp. and Fusarium spp. (Baker, 1989 and 
Chet, 1987). The Arbuscular mycorrhizal fungi (AMF) can stimulate plant growth especially in soils with low fertility mainly due to improved phosphorous absorption (Johnson, et al (1982) and Smith, et al (1986)). Arbuscular mycorrhizal fungi have been reported to protect plant roots from some root infecting fungi (Caron, 1989). This study was carried out to investigate the effects AMF and $T$. harzianum in enhancing growth and controlling wilt tomato caused by $F$. oxysporum f. sp. lycopersici.

\section{Material and Methods}

\section{Source of the pathogenic fungi:}

Pathogenic fungal isolate of Fusarium oxysporum f. sp. lycopersici were isolated from roots of tomato plants (Peto 86 variety) were obtained from Fac. of Agric. Ain Shams University, plants had shown signs of wilting and had a brown discoloration of vascular vessels, fungus isolated and identified according to method described by Nelson et al. (1983).

F. oxysporum f. sp. lycopersici was grown in darkness at $28^{\circ} \mathrm{C}$ on Potato Dextrose Agar (PDA) plates till full sporlation. Then, spores were transferred with sterilized distilled water and arriving to $10^{7}$ conidia $/ \mathrm{ml}$.

The sterilized soils in plastic pots $(30 \mathrm{~cm})$ were infested with this isolate of Fusarium at a concentration of $10^{7}$ conidia/ $\mathrm{ml}$ of the substrate $(40 \mathrm{ml} / \mathrm{pot})$ and was allowed to establish in the soil for a period of a week.

Source and Preparation of Trichoderma harzianum inoculum:

Fungal isolate of $T$. harzianum was obtained from tomato rhizosphere and field soil during the preliminary study according to methods described by Elad and Chet (1983) and Harman (2006) and identified according to Rifai (1969).

Trichoderma harzianum was grown in darkness at $28^{\circ} \mathrm{C}$ on Potato Dextrose Agar (PDA) plates till the colony became green. Then, spores were transferred with sterilized distilled water and arriving to $10^{7}$ spores $/ \mathrm{ml}$ of the conidial suspension concentration. The root systems of tomato seedlings were washed in tap water then immersed in a conidial suspension and then transplanted into plastic pots $(30 \mathrm{~cm})$.

\section{Source of AMF inoculum}

For AMF inoculation, spore suspension from Microbiological Researches Center (Cairo Mircen) Egypt Microbiological Culture Collection (EMCC) Fac. of Agr. Ain Shams Univ. was utilized as a commercially available inoculum. This AMF inoculum holds at least 80,000 spores/liter and includes two different species of AMF (Glomus microagregatum and Glomus claroideum,) (Hage-Ahmed et al., 2013).

\section{Preparation of tomato seedling:}

Certified tomato seeds of three cultivars; Castle-rock, Super Marmande and Peto 86 were surface sterilized in $1 \%$ solution of sodium hypochlorite for $30 \mathrm{sec}$ and rinsed thoroughly with several changes of distilled water and then dried with sterile blotting paper. The seeds were then germinated in $15 \times 20 \times 10 \mathrm{~cm}$ trays containing sterilized sand. Twenty eight day old tomato seedlings were transplanted into plastic pots $(30 \mathrm{~cm}$ diameter), at the rate of three plants per pot.

Experiment Design: 
Effect of $T$. harzianum and AMF on growth and disease control of fusarium wilt has been tested in five treatments applied as follows; $T$. harzianum, AMF (Arbuscular mycorrhizal fungi), AMF $+T$. harzianum, control (1) (contained only the pathogen $F$. oxysporum f. sp. lycopersiciontrol) and control (2) in autoclaved non infested soil. Each treatment was replicated 5 times and placed in the green house in a completely randomized design. Plants were watered daily.

Measurements:

After 8 weeks; disease severity, height, shoot and root fresh and dry weights were determined.

The shoots and the roots were dried in an oven at $70{ }^{\circ} \mathrm{C}$ until constant weight, then weighed separately and the weights recorded.

Disease Severity (DS) assessment was done when symptoms of infection were observed. Such symptoms included clearing of the veins and drooping of petioles followed by yellowing of lower leaves (Agrios, 1988). Wilt severity was determined using a scale by Waudo et al. (1995). This was based on the wilt severity rated as follows; (\% of shoot wilted, using a scale of $0-5$ where, $0=$ No symptoms, $1=$ One leaf wilted $(1 \%-25 \%), 2=2$ or 3 leaves wilted $(26 \%-49 \%), \quad 3=$ half plant wilted $(50 \%-74 \%), \quad 4=$ all leaves wilted (75\%-100\%), 5=Plant dead).

\section{Chemical analysis:}

\section{Determination of free Amino acids}

Free amino acids were determined colourimetrically by using ninhydrin solution according to Jayeraman (1985) using glycine as a standard. The amino acids were calculated as $\mathrm{mg} / 100 \mathrm{~g}$ f.wt.

\section{Determination of Reducing sugers}

Reducing sugers were determined colourimetrically by using 3,5 dinitrosalsylic acid solution according to Miller (1959) using glucose as a standard. The reducing sugers were calculated as $\mathrm{mg} / 100 \mathrm{~g}$ f.wt.

Determination of phenolic compounds

The colorimetric method of Folin-Ciocalteu as described by Shahidi and Naczk, (1995) was employed for the chemical determination of phenolic compounds.The phenolic compounds concentration was expressed as mg gallic acid / $100 \mathrm{~g}$ f.wt.

\section{Determination of soluble protein}

Soluble protein concentration was estimated to calculate specific activity of enzymes. Proteins concentration was quantified in the crude extract by the method of Bradford (1976) using bovine serum albumin as a standard.

\section{Enzymes assay \\ Preparation of enzymes crude extract}

Tissue were homogenized with potassium phosphate buffer (100 $\mathrm{mM}, \mathrm{pH}=7.0$ ) containing $0.1 \mathrm{mM}$ EDTA and $1 \%$ polyvinyl pyrrolidone $(\mathrm{PVP})(\mathrm{W} / \mathrm{V})$ at $4^{\circ} \mathrm{C}$. The extraction ratio was $4 \mathrm{ml}$ buffer for each one gram of plant materials. Homogenate was centrifuged at $15000 \times \mathrm{g}$ for 15 $\min$ at $4^{\circ} \mathrm{C}$. Supernatant was considered as enzyme crude extract and used to measure the activities of guaiacol peroxidase (POD), catalase, polyphenol oxidase (PPO), phenylalanine ammonia lyase (PAL) and ascorbate peroxidase.

Assays of enzymes: 
1- peroxidase activity

Peroxidase, POD (E.C 1.11.1.7) activity in enzyme crude extract was determined as described by Hammer Schmidt et al. (1982). The activity was calculated by measuring the absorbance changes at $470 \mathrm{~nm}$ per min using spectrophotometer (UV-Vis spectrophotometer UV $9100 \mathrm{~B}$, LabTech). Unit of enzyme (IU) equal $0.01 \Delta$ OD. min-1. The specific activity expressed as unit/ $\mathrm{mg}$ protein.

\section{activity}

2- Polyphenol oxidase (PPO)

Polyphenol oxidase (PPO) (EC 1.14.18.1) activity was measured according to Benjamin and Montegomery (1973). One unit of PPO activity was defined as the amount of enzyme that caused an increase in absorbance of 0.001 per min at $420 \mathrm{~nm}$ using spectrophotometer (UV-Vis spectrophotometer UV $9100 \mathrm{~B}$, LabTech). The enzyme activity was expressed as unit/mg protein.

\section{3- Phenylalanine ammonia} lyase (PAL) activity

Phenylalanine ammonia lyase (PAL) (EC 4.3.1.5) activity was quantified by the method of Beaudoin-Eagan and Thorpe (1985). One unit of enzyme activity was defined as the amount of enzyme that caused an increase in absorbance of 0.01 per hour at $290 \mathrm{~nm}$ using spectrophotometer (UV-Vis spectrophotometer UV 9100 B, LabTech). The enzyme activity was expressed as unit/mg protein.

4- Ascorbate peroxidase (APX) activity

Ascorbate peroxidase (APX) (E.C 1.11.1.11) was measured according to Nakano and Asada (1981). One unit of enzyme activity was de- fined as the amount of enzyme required for oxidation of $1 \mu \mathrm{mol}$ of ascorbate per minute and caused a decrease in absorbance at $290 \mathrm{~nm}$ using spectrophotometer (UV-Vis spectrophotometer UV 9100 B, LabTech). The enzyme activity was expressed as unit/mg protein.

\section{Statistical analysis}

All data were subjected to one way analysis of variance (ANOVA) followed by means separation through least significant difference (L.S.D.) test at $\mathrm{P}<0.05$ level(Snedecor and Cochran, 1980).

\section{Results and Discussion \\ Effect of treatments on wilt patho- gen control}

Data in Table (1) indicated that tomato wilt disease severity was reduced significantly by all treatments in the three varieties, individually the biocontrol fungus $T$. harzianum and AMF as compared to the control (infested) where the biocontrol fungi had not been added, on the other hand, there was no significant differences in reduction of disease severity between the individually treatments.

A combination of T. harzianum and AMF had less trend of severity of disease in Castle-rock, Super Marmande and Peto 86 varieties which disease severity were (6.67, 2.23 and $11.10 \%$ ) compared to control infested soil $(77.7,68.90$ and $82.20 \%$ ) respectively, in dependent on this results $\mathrm{AMF}+T$. harzianum treatment efficiency was increased $(91.42,96,76$ and $85.87 \%)$ respectively in the three varieties compared to control infested.

In an experiment where commercial formulations of AMF and $T$. harzianum were applied to control 
fusarium wilt, each of the fungi showed significant disease control but better results were obtained when both agents were used together (Datnoff, et al 1995).

The ability of $T$. harzianum to control plant pathogens is considered to be a result of several mechanisms including mycoparasitism and induction of systematic defense mechanism (Chet, (1987) and Yedida, et al (1999)). On the other hand, AMF has been known to increase plant resistance to infection through improved plant nutrition (Declerck, et al 2002).

A comparison of mycorrhizal root colonization in the treatment
AMF and treatment with both AMF and $T$. harzianum showed that they were not significantly different (Mwangi, et al (2011)), also they demonstrated that $T$. harzianum showed compatibility with AMF and no inhibitory effects on the development of mycorrhizal colonization was observed, also Sr'amek, et al 2000 found that $T$. harzianum did not affect colonization by AMF in three balcony plants (Verbena, Torenia, Diascia) inoculated with both AMF and T. harzianum.

Table 1. Effect of biological treatments on tomato wilt disease severity (DS) and the efficiency after 8 weeks on three varieties of tomato.

\begin{tabular}{|c|c|c|c|c|c|c|}
\hline \multirow{2}{*}{ Treatment } & \multicolumn{2}{|c|}{ Castle-rock } & \multicolumn{2}{|c|}{ Super Marmande } & \multicolumn{2}{|c|}{ Peto 86} \\
\hline & $\begin{array}{c}\text { \%Disease } \\
\text { severity }\end{array}$ & $\begin{array}{c}\% \\
\text { efficiency }\end{array}$ & $\begin{array}{c}\text { \%Disease } \\
\text { severity }\end{array}$ & $\begin{array}{c}\% \\
\text { efficiency }\end{array}$ & $\begin{array}{c}\text { \%Disease } \\
\text { severity }\end{array}$ & $\begin{array}{c}\% \\
\text { efficiency }\end{array}$ \\
\hline $\mathbf{A M F}$ & $\begin{array}{c}13.33 \mathrm{CB} \\
\pm 5.43\end{array}$ & 82.86 & $\begin{array}{c}4.47 \mathrm{CB} \\
\pm 3.16\end{array}$ & 93.51 & $\begin{array}{c}26.67 \mathrm{CB} \\
\pm 5.43\end{array}$ & 67.07 \\
\hline T. harzianum & $\begin{array}{c}20.00 \mathrm{~B} \\
\pm 5.47\end{array}$ & 74.28 & $\begin{array}{l}17.77 \mathrm{~B} \\
\pm 6.32\end{array}$ & 74.21 & $\begin{array}{c}28.90 \mathrm{~B} \\
\pm 3.11\end{array}$ & 64.37 \\
\hline $\begin{array}{l}\mathrm{AMF}+T . \\
\text { harzianum }\end{array}$ & $\begin{array}{c}6.67 \mathrm{CB} \\
\pm 5.43\end{array}$ & 91.42 & $\begin{array}{c}2.23 \mathrm{CB} \\
\pm 3.16\end{array}$ & 96.76 & $\begin{array}{c}11.10 \mathrm{CD} \\
\pm 3.11 \\
\end{array}$ & 85.87 \\
\hline $\begin{array}{c}\text { Control } \\
\text { (autoclaved soil) }\end{array}$ & $\begin{array}{l}0.00 \mathrm{C} \\
\pm 0.00\end{array}$ & 100.00 & $\begin{array}{l}0.00 \mathrm{C} \\
\pm 0.00\end{array}$ & 100.00 & $\begin{array}{l}0.00 \mathrm{D} \\
\pm 0.00\end{array}$ & 100.00 \\
\hline Control (infested soil) & $\begin{array}{l}77.77 \mathrm{~A} \\
\pm 3.16\end{array}$ & 0.00 & $\begin{array}{c}68.90 \mathrm{~A} \\
\pm 8.31\end{array}$ & 0.00 & $\begin{array}{c}82.20 \mathrm{~A} \\
\pm 8.32\end{array}$ & 0.00 \\
\hline & 14.635 & & 1.9652 & & 2.0983 & \\
\hline
\end{tabular}

Data presented as the means of three replicates \pm SD. Different letters refer to significant difference $(\mathrm{P} \leq 0.05)$.

\section{Effect of treatments on growth pa- rameters:}

Data in Table (2 and 3) and Figure (1) indicated that all treatments enhanced morphological parameters significantly compared to control infested soil. The T. harzianum or AMF applied singly enhanced root parame- ters significantly compared to the control (Table 3). Observed significant growth due to mycorrhizal infection in tomatoes confirms other reports that AMF symbiosis with host plant has an improved growth effect (Dubsk, et al (2002) and Sr'amek, et al (2000)). Increased growth due to 
mycorrhizal infection is mainly attributed to improved phosphorous and micronutrient uptake in the host plant (Johnson, et al (1982) and Smith, et al (1986)). These results also agree with those of Ozbay, and Newman, (2004) where T. harzianum strains were also reported to have significantly increased the height, shoot and root dry weight in tomato seedlings transplanted into pots in the green house. There are reports that $T$. harzianum increases the solubility of phosphates and micronutrients such as zinc, copper, iron and manganese all plant nutrients with low solubility (Altomare, et al 1999) and this enhances growth of the roots and the above ground parts of the plant.

The treatment with a combination of the two fungi (T. harzianum and AMF) also highly significant enhanced growth more than all treatment in Castle-rock, Super Marmande and Peto 86 varieties compared to control infested soil. Dual inoculation with $T$. harzianum and AMF has previously been reported to significantly enhance growth more than if each fungus was inoculated singly (Dubsk, et al (2002) and Sr'amek, et al (2000)). Some strains of $T$. harzianum establish robust and long lasting colonization of root surfaces penetrating into the epidermis (Harman, 2000), this colonization by $T$. harzianum frequently enhances root growth development, crop productivity and resistance to abiotic stresses through enhancement of mineral absorption. 
Table 2. Effect of biological treatments on shoot mean height, fresh and dry weight and \% dry weight of three varieties of tomato after 8 weeks.

\begin{tabular}{|c|c|c|c|c|c|c|c|c|c|c|c|c|}
\hline \multirow[b]{2}{*}{ Treatment } & \multicolumn{4}{|c|}{ Castle-rock } & \multicolumn{4}{|c|}{ Super Marmande } & \multicolumn{4}{|c|}{ Peto 86} \\
\hline & $\begin{array}{c}\text { shoot } \\
\text { height } \\
(\mathrm{cm})\end{array}$ & $\begin{array}{c}\text { shoot } \\
\text { fresh } \\
\text { weight } \\
\text { (gm) }\end{array}$ & $\begin{array}{c}\text { shoot } \\
\text { dry } \\
\text { weight } \\
(\mathrm{gm})\end{array}$ & $\begin{array}{c}\% \\
\text { Dry } \\
\text { weight }\end{array}$ & $\begin{array}{c}\text { shoot } \\
\text { height } \\
\text { (cm) }\end{array}$ & $\begin{array}{c}\text { shoot } \\
\text { fresh } \\
\text { weight } \\
(\mathrm{gm})\end{array}$ & $\begin{array}{c}\text { shoot } \\
\text { dry } \\
\text { weight } \\
\text { (gm) }\end{array}$ & $\begin{array}{c}\% \\
\text { Dry } \\
\text { weight }\end{array}$ & $\begin{array}{c}\text { shoot } \\
\text { height } \\
(\mathrm{cm})\end{array}$ & $\begin{array}{c}\text { shoot } \\
\text { fresh } \\
\text { weight } \\
(\mathrm{gm})\end{array}$ & $\begin{array}{c}\text { shoot } \\
\text { dry } \\
\text { weight } \\
\text { (gm) }\end{array}$ & $\begin{array}{c}\text { \% } \\
\text { Dry } \\
\text { weight }\end{array}$ \\
\hline & & & & & & & & & & & & \\
\hline & & & & & & & & & & & & \\
\hline & $\begin{array}{r}61.50 \\
\pm 0.4 \\
\end{array}$ & $\begin{array}{c}16.67 \mathrm{~A} \\
\pm 0.41\end{array}$ & $\begin{array}{l}2.70 \mathrm{~A} \\
\pm 0.08\end{array}$ & & & & & & & $\begin{array}{c}28.47 \mathrm{~A} \\
\pm 0.41\end{array}$ & $\begin{array}{l}4.20 \mathrm{~A} \\
\pm 0.08\end{array}$ & \\
\hline $\begin{array}{l}\text { ol } \\
\text { aved }\end{array}$ & $\begin{array}{c}35.17 \mathrm{D} \\
\pm 0.85\end{array}$ & $\begin{array}{c}11.03 \mathrm{C} \\
\pm 0.86\end{array}$ & $\begin{array}{l}0.98 \mathrm{C} \\
\pm 0.05\end{array}$ & & & $\begin{array}{c}16.57 \mathrm{D} \\
\pm 1.56\end{array}$ & & & & $\begin{array}{l}8.87 \mathrm{D} \\
\pm 0.66\end{array}$ & $\begin{array}{l}0.81 \mathrm{D} \\
\pm 0.02\end{array}$ & \\
\hline $\begin{array}{c}\text { Control } \\
\text { (infested } \\
\text { soil) }\end{array}$ & $\begin{array}{c}26.87 \mathrm{E} \\
\pm 0.66\end{array}$ & $\begin{array}{l}7.50 \mathrm{D} \\
\pm 0.41\end{array}$ & $\begin{array}{l}0.53 \mathrm{D} \\
\pm 0.05\end{array}$ & & & $\begin{array}{c}16.17 \mathrm{D} \\
\pm 0.24\end{array}$ & $\begin{array}{l}1.27 \mathrm{C} \\
\pm 0.05\end{array}$ & $\begin{array}{l}7.83 \mathrm{~B} \\
\pm 0.26\end{array}$ & & $\begin{array}{l}4.10 \mathrm{E} \\
\pm 0.43\end{array}$ & $\begin{array}{l}0.30 \mathrm{E} \\
\pm 0.01\end{array}$ & $\begin{array}{l}7.38 \mathrm{l} \\
\pm 0.6\end{array}$ \\
\hline & & & & & & & & & & & & \\
\hline
\end{tabular}

Data presented as the means of three replicates \pm SD. Different letters refer to significant difference $(\mathrm{P} \leq$ $0.05)$.

Table 3. Effect of biological treatments on root mean length, fresh and dry weight and \% dry weight of three varieties of tomato after 8 weeks.

\begin{tabular}{|c|c|c|c|c|c|c|c|c|c|c|c|c|}
\hline \multirow[b]{2}{*}{ Yariety } & \multicolumn{4}{|c|}{ Castle-rock } & \multicolumn{4}{|c|}{ Super Marmande } & \multicolumn{4}{|c|}{ Peto 86} \\
\hline & $\begin{array}{l}\text { Root } \\
\text { Length } \\
\text { (cm) }\end{array}$ & \begin{tabular}{|c|} 
Root \\
fresh \\
weight \\
(gm)
\end{tabular} & \begin{tabular}{|c|} 
Root \\
dry \\
weight \\
(gm) \\
\end{tabular} & $\begin{array}{l}\% \text { Dry } \\
\text { weight }\end{array}$ & $\begin{array}{c}\text { Root } \\
\text { Length } \\
\text { (cm) }\end{array}$ & \begin{tabular}{|c|}
$\begin{array}{c}\text { Root } \\
\text { fresh } \\
\text { weight } \\
\text { (gm) }\end{array}$ \\
\end{tabular} & \begin{tabular}{|c|} 
Root \\
dry \\
weight \\
$($ gm)
\end{tabular} & $\begin{array}{c}\text { \% } \\
\text { Dry } \\
\text { weight }\end{array}$ & $\begin{array}{c}\text { Root } \\
\text { Length } \\
\text { (cm) }\end{array}$ & \begin{tabular}{|c|} 
Root \\
fresh \\
weight \\
(gm)
\end{tabular} & \begin{tabular}{|c|}
$\begin{array}{c}\text { Root } \\
\text { dry } \\
\text { weight } \\
\text { (gm) }\end{array}$ \\
\end{tabular} & $\begin{array}{c}\% \\
\text { Dry } \\
\text { weight }\end{array}$ \\
\hline AMF & $\begin{array}{c}29.67 \mathrm{~B} \\
\pm 0.47\end{array}$ & $\begin{array}{l}5.87 \mathrm{~A} \\
\pm 0.26\end{array}$ & $\begin{array}{l}0.73 \mathrm{~A} \\
\pm 0.05\end{array}$ & $\begin{array}{c}12.43 \mathrm{BA} \\
\pm 0.30\end{array}$ & $\begin{array}{c}37.43 \mathrm{~B} \\
\pm 0.42\end{array}$ & $\begin{array}{c}11.07 \mathrm{~B} \\
\pm 0.74 \\
\end{array}$ & $\begin{array}{l}1.40 \mathrm{~B} \\
\pm 0.08\end{array}$ & $\begin{array}{c}12.68 \mathrm{BA} \\
\pm 0.81\end{array}$ & $\begin{array}{c}32.23 \mathrm{~A} \\
\pm 0.56\end{array}$ & $\begin{array}{l}6.97 \mathrm{~B} \\
\pm 0.53\end{array}$ & $\begin{array}{l}0.87 \mathrm{~B} \\
\pm 0.06\end{array}$ & $\begin{array}{c}12.55 \mathrm{~B} \\
\pm 0.14\end{array}$ \\
\hline $\begin{array}{c}\text { T. har- } \\
\text { zianum }\end{array}$ & $\begin{array}{c}27.10 \mathrm{C} \\
\pm 0.70 \\
\end{array}$ & $\begin{array}{l}5.97 \mathrm{~A} \\
\pm 0.21 \\
\end{array}$ & \begin{tabular}{|l|}
$0.75 \mathrm{~A}$ \\
\pm 0.03 \\
\end{tabular} & \begin{tabular}{c|}
$12.62 \mathrm{~A}$ \\
\pm 0.10 \\
\end{tabular} & \begin{tabular}{|c|}
$37.67 \mathrm{BA}$ \\
\pm 0.47 \\
\end{tabular} & $\begin{array}{c}13.66 \mathrm{~A} \\
\pm 0.30 \\
\end{array}$ & \begin{tabular}{|l|}
$1.74 \mathrm{~A}$ \\
\pm 0.04 \\
\end{tabular} & $\begin{array}{c}12.74 \mathrm{BA} \\
\pm 0.08 \\
\end{array}$ & $\begin{array}{c}32.67 \mathrm{~A} \\
\pm 0.47 \\
\end{array}$ & \begin{tabular}{|l|}
$8.13 \mathrm{~A}$ \\
\pm 0.26 \\
\end{tabular} & \begin{tabular}{|l|}
$1.23 \mathrm{~A}$ \\
\pm 0.12 \\
\end{tabular} & $\begin{array}{c}15.13 \mathrm{~A} \\
\pm 1.04 \\
\end{array}$ \\
\hline $\begin{array}{l}\text { AMF + T. } \\
\text { harzianum }\end{array}$ & $\begin{array}{c}33.17 \mathrm{~A} \\
\pm 0.85\end{array}$ & $\begin{array}{l}6.50 \mathrm{~A} \\
\pm 0.24 \\
\end{array}$ & \begin{tabular}{|l|}
$0.75 \mathrm{~A}$ \\
\pm 0.02 \\
\end{tabular} & $\begin{array}{c}11.59 \mathrm{BC} \\
\pm 0.09 \\
\end{array}$ & $\begin{array}{c}39.57 \mathrm{~A} \\
\pm 0.37 \\
\end{array}$ & $\begin{array}{c}11.83 \mathrm{~B} \\
\pm 0.41 \\
\end{array}$ & \begin{tabular}{|l|}
$1.73 \mathrm{~A}$ \\
\pm 0.12 \\
\end{tabular} & $\begin{array}{c}14.63 \mathrm{~A} \\
\pm 0.58 \\
\end{array}$ & $\begin{array}{c}32.93 \mathrm{~A} \\
\pm 0.42 \\
\end{array}$ & \begin{tabular}{|c|}
$7.83 \mathrm{BA}$ \\
\pm 0.41 \\
\end{tabular} & \begin{tabular}{l|}
$1.27 \mathrm{~A}$ \\
\pm 0.12 \\
\end{tabular} & $\begin{array}{c}16.13 \mathrm{~A} \\
\pm 0.77\end{array}$ \\
\hline $\begin{array}{c}\begin{array}{c}\text { Control } \\
\text { (autoclaved } \\
\text { soil) }\end{array} \\
\end{array}$ & $\begin{array}{c}29.00 \mathrm{CB} \\
\pm 0.82\end{array}$ & $\begin{array}{l}3.47 \mathrm{~B} \\
\pm 0.27 \\
\end{array}$ & $\begin{array}{l}0.39 \mathrm{~B} \\
\pm 0.02\end{array}$ & $\begin{array}{c}11.38 \mathrm{C} \\
\pm 0.47\end{array}$ & $\begin{array}{c}31.10 \mathrm{C} \\
\pm 0.94\end{array}$ & $\begin{array}{l}6.23 \mathrm{C} \\
\pm 0.17 \\
\end{array}$ & $\begin{array}{l}0.67 \mathrm{C} \\
\pm 0.01\end{array}$ & $\begin{array}{c}10.78 \mathrm{BC} \\
\pm 0.16\end{array}$ & $\begin{array}{c}19.10 \mathrm{~B} \\
\pm 0.94 \\
\end{array}$ & $\begin{array}{l}4.30 \mathrm{C} \\
\pm 0.16 \\
\end{array}$ & $\begin{array}{l}0.45 \mathrm{C} \\
\pm 0.02 \\
\end{array}$ & $\begin{array}{c}10.48 \mathrm{CB} \\
\pm 0.20\end{array}$ \\
\hline $\begin{array}{c}\text { Control } \\
\text { (infested } \\
\text { soil) }\end{array}$ & $\begin{array}{c}16.33 \mathrm{D} \\
\pm 0.47\end{array}$ & $\begin{array}{l}2.47 \mathrm{C} \\
\pm 0.12 \\
\end{array}$ & $\begin{array}{l}0.24 \mathrm{C} \\
\pm 0.01 \\
\end{array}$ & $\begin{array}{l}9.74 \mathrm{D} \\
\pm 0.19\end{array}$ & $\begin{array}{c}14.00 \mathrm{D} \\
\pm 0.82\end{array}$ & $\begin{array}{l}3.77 \mathrm{D} \\
\pm 0.29 \\
\end{array}$ & $\begin{array}{l}0.38 \mathrm{D} \\
\pm 0.01 \\
\end{array}$ & $\begin{array}{c}10.24 \mathrm{C} \\
\pm 0.88\end{array}$ & $\begin{array}{c}13.33 \mathrm{C} \\
\pm 0.47\end{array}$ & $\begin{array}{l}2.23 \mathrm{D} \\
\pm 0.09\end{array}$ & $\begin{array}{l}0.21 \mathrm{C} \\
\pm 0.01 \\
\end{array}$ & $\begin{array}{l}9.42 \mathrm{C} \\
\pm 0.54\end{array}$ \\
\hline L.S.D. & 2.2418 & 0.7632 & 0.0961 & 0.8892 & 2.1261 & 1.4055 & 0.2263 & 1.9652 & 1.9832 & 1.097 & 0.2753 & 2.0983 \\
\hline
\end{tabular}

Data presented as the means of three replicates \pm SD. Different letters refer to significant difference (P $\leq$ 0.05 ). 
Table 3. Effect of biological treatments on root mean length, fresh and dry weight and \% dry weight of three varieties of tomato after 8 weeks.

\begin{tabular}{|c|c|c|c|c|c|c|c|c|c|c|c|c|}
\hline \multirow[b]{2}{*}{ Variety } & \multicolumn{4}{|c|}{ Castle-rock } & \multicolumn{4}{|c|}{ Super Marmande } & \multicolumn{4}{|c|}{ Peto 86} \\
\hline & $\begin{array}{l}\text { Root } \\
\text { Length } \\
\text { (cm) }\end{array}$ & $\begin{array}{c}\text { Root } \\
\text { fresh } \\
\text { weight } \\
(\text { gm) }\end{array}$ & \begin{tabular}{|c} 
Root \\
dry \\
weight \\
$(\mathrm{gm})$
\end{tabular} & $\begin{array}{l}\text { \% Dry } \\
\text { weight }\end{array}$ & $\begin{array}{l}\text { Root } \\
\text { Length } \\
\text { (cm) }\end{array}$ & $\begin{array}{c}\text { Root } \\
\text { fresh } \\
\text { weight } \\
(\mathrm{gm})\end{array}$ & $\begin{array}{c}\begin{array}{c}\text { Root } \\
\text { dry } \\
\text { weight } \\
(\mathrm{gm})\end{array} \\
\end{array}$ & $\begin{array}{l}\% \text { Dry } \\
\text { weight }\end{array}$ & $\begin{array}{c}\text { Root } \\
\text { Length } \\
\text { (cm) }\end{array}$ & $\begin{array}{c}\text { Root } \\
\text { fresh } \\
\text { weight } \\
(\text { gm) }\end{array}$ & $\begin{array}{c}\text { Root } \\
\text { dry } \\
\text { weight } \\
\text { (gm) }\end{array}$ & $\begin{array}{c}\% \\
\text { Dry } \\
\text { weight }\end{array}$ \\
\hline AMF & $\begin{array}{c}29.67 \mathrm{~B} \\
\pm 0.47\end{array}$ & $\begin{array}{l}5.87 \mathrm{~A} \\
\pm 0.26\end{array}$ & & & & & & & & & & \\
\hline $\begin{array}{c}\text { T. har- } \\
\text { zianum }\end{array}$ & $\begin{array}{c}27.10 \mathrm{C} \\
\pm 0.70\end{array}$ & $\begin{array}{l}5.97 \mathrm{~A} \\
\pm 0.21\end{array}$ & $\begin{array}{l}0.75 \mathrm{~A} \\
\pm 0.03\end{array}$ & $\begin{array}{c}12.62 \mathrm{~A} \\
\pm 0.10\end{array}$ & $\begin{array}{c}37.67 \mathrm{BA} \\
\pm 0.47\end{array}$ & $\begin{array}{c}13.66 \mathrm{~A} \\
\pm 0.30\end{array}$ & $\begin{array}{l}1.74 \mathrm{~A} \\
\pm 0.04\end{array}$ & $\begin{array}{c}12.74 \mathrm{BA} \\
\pm 0.08\end{array}$ & $\begin{array}{c}32.67 \mathrm{~A} \\
\pm 0.47\end{array}$ & $\begin{array}{l}8.13 \mathrm{~A} \\
\pm 0.26\end{array}$ & $\begin{array}{l}1.23 \mathrm{~A} \\
\pm 0.12\end{array}$ & $\begin{array}{c}15.13 \mathrm{~A} \\
\pm 1.04\end{array}$ \\
\hline $\begin{array}{l}\text { AMF + T. } \\
\text { harzianum }\end{array}$ & $\begin{array}{c}33.17 \mathrm{~A} \\
\pm 0.85\end{array}$ & $\begin{array}{l}6.50 \mathrm{~A} \\
\pm 0.24 \\
\end{array}$ & & & $\begin{array}{c}39.57 \mathrm{~A} \\
\pm 0.37 \\
\end{array}$ & & $\begin{array}{l}1.73 \mathrm{~A} \\
\pm 0.12 \\
\end{array}$ & & $\begin{array}{c}32.93 \mathrm{~A} \\
\pm 0.42 \\
\end{array}$ & $\begin{array}{c}7.83 \mathrm{BA} \\
\pm 0.41\end{array}$ & $\begin{array}{l}1.27 \mathrm{~A} \\
\pm 0.12\end{array}$ & $\begin{array}{c}16.13 \mathrm{~A} \\
\pm 0.77\end{array}$ \\
\hline $\begin{array}{c}\begin{array}{c}\text { Control } \\
\text { (autoclaved } \\
\text { soil) }\end{array} \\
\end{array}$ & $\begin{array}{c}29.00 \mathrm{CB} \\
\pm 0.82\end{array}$ & $\begin{array}{l}3.47 \mathrm{~B} \\
\pm 0.27\end{array}$ & $\begin{array}{l}0.39 \mathrm{~B} \\
\pm 0.02\end{array}$ & $\begin{array}{c}11.38 \mathrm{C} \\
\pm 0.47\end{array}$ & $\begin{array}{c}31.10 \mathrm{C} \\
\pm 0.94\end{array}$ & $\begin{array}{l}6.23 \mathrm{C} \\
\pm 0.17\end{array}$ & $\begin{array}{l}0.67 \mathrm{C} \\
\pm 0.01\end{array}$ & $\begin{array}{c}10.78 \mathrm{BC} \\
\pm 0.16\end{array}$ & $\begin{array}{c}19.10 \mathrm{~B} \\
\pm 0.94\end{array}$ & $\begin{array}{l}4.30 \mathrm{C} \\
\pm 0.16\end{array}$ & $\begin{array}{l}0.45 \mathrm{C} \\
\pm 0.02\end{array}$ & $\begin{array}{c}10.48 \mathrm{CB} \\
\pm 0.20\end{array}$ \\
\hline $\begin{array}{c}\text { Control } \\
\text { (infested } \\
\text { soil) }\end{array}$ & $\begin{array}{c}16.33 \mathrm{D} \\
\pm 0.47\end{array}$ & $\begin{array}{l}2.47 \mathrm{C} \\
\pm 0.12\end{array}$ & $\begin{array}{l}0.24 \mathrm{C} \\
\pm 0.01\end{array}$ & & $\begin{array}{c}14.00 \mathrm{D} \\
\pm 0.82\end{array}$ & $\begin{array}{l}3.77 \mathrm{D} \\
\pm 0.29\end{array}$ & $\begin{array}{l}0.38 \mathrm{D} \\
\pm 0.01\end{array}$ & $\begin{array}{c}10.24 \mathrm{C} \\
\pm 0.88\end{array}$ & $\begin{array}{c}13.33 \mathrm{C} \\
\pm 0.47\end{array}$ & $\begin{array}{l}2.23 \mathrm{D} \\
\pm 0.09\end{array}$ & $\begin{array}{l}0.21 \mathrm{C} \\
\pm 0.01\end{array}$ & $\begin{array}{l}9.42 \mathrm{C} \\
\pm 0.54\end{array}$ \\
\hline L.S.D. & 2.2418 & 0.7632 & 0.0961 & 0.8892 & 1261 & 1.4055 & 0.2263 & 1.9652 & 1.9832 & 1.097 & 0.2753 & 2.0983 \\
\hline
\end{tabular}

Data presented as the means of three replicates $\pm \mathrm{SD}$. Different letters refer to significant difference $(\mathrm{P} \leq 0.05)$.

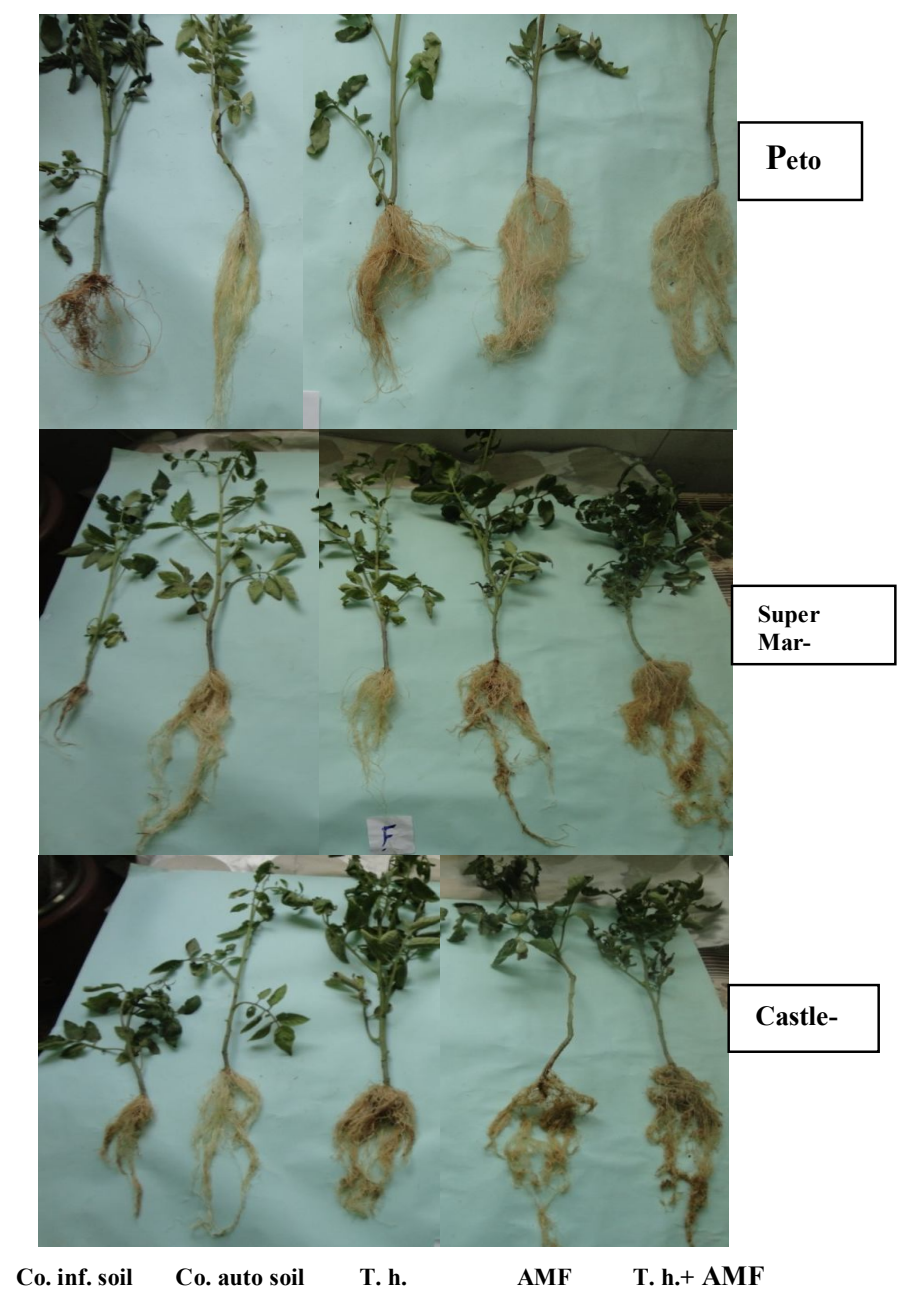

Fig. 1. Effect of biological treatments on morphological parameters of three varieties of tomato. 


\section{Biochemical changes:}

There were significant differences in phenolic compounds, amin acids and reducing sugars contents in all treatments (Table 4). AMF $+T$. harzianum treatment showed the highest phenolic compounds content in tomato roots of all varieties, where $(146.85,65.71$ and $116.61 \mathrm{mg} / 100 \mathrm{~g}$ f.wt.) in castle-rock, super marmande and peto 86 respectively, followed by $T$. harzianum treatment in comparison with control infected and healthy control. While AMP treatment the lowest value 44.57, 23.36 and 44.19 $\mathrm{mg} / 100 \mathrm{~g}$ f.wt. in castle-rock, super marmande and peto 86 respectively.

Also amino acids had the same trend of phenolic compounds under influene of $\mathrm{AMF}+T$. harzianum and $T$. harzianum treatments which gave the highest content in all varieties of tomato roots (Table 4), followed by AMF treatment. Reducing sugars had the same trend of phenolic com- pounds and amino acids in peto 86 roots (Table 4). While reducing sugars were higher in AMF $+T$. harzianum followed by AMF and T. harzianum treatment which gave the lower value (102.47 and 183.88 $\mathrm{mg} / 100 \mathrm{~g}$ f.wt.) in castle-rock, super marmande varieties in comparision with infected control (141.72 and $188.17 \mathrm{mg} / 100 \mathrm{~g}$ f.wt ) respectively.

There were significant differences in phenolic compounds, amino acids and reducing sugars contents in all treatments (Table 5). phenolic compounds content had the highest value in tomato leaves under influence of $\mathrm{AMF}+T$. harzianum treatment in $(187.05,184.64$ and 157.25 $\mathrm{mg} / 100 \mathrm{~g}$ f.wt.) in castle-rock, super marmande and peto 86 respectively, while T. harzianum and AMF treatments gave lower content in comparison with infected control in all varieties .

Table 4. Effect of AMF, T. harzianum and AMF + T. harzianum biological treatments on roots content of Phenols, Amino acids and Reducing sugars after 8 weeks in three varieties of tomato infected with Fusarium oxysporum f. sp. lycopersici.

\begin{tabular}{|c|c|c|c|c|c|c|c|c|c|}
\hline \multirow{2}{*}{ Variety } & \multicolumn{3}{|c|}{ Castle-rock } & \multicolumn{3}{|c|}{ Super Marmande } & \multicolumn{3}{|c|}{ Peto 86} \\
\hline & Phenols & & & Phenols & & & Phenols & & \\
\hline & & & & & & & & & \\
\hline $\begin{array}{l}\text { T. har- } \\
\text { zianum }\end{array}$ & & & & & & & & & \\
\hline & & & & & & & & & \\
\hline $\begin{array}{c}\text { Control } \\
\text { (autoclaved }\end{array}$ & $\begin{array}{c}36.01 \mathrm{E} \\
\pm 1.81\end{array}$ & 101.30B & $\begin{array}{c}161.74 \mathrm{C} \\
\pm 1.15\end{array}$ & $\begin{array}{c}40.89 \mathrm{C} \\
\pm 1.32\end{array}$ & $\begin{array}{c}123.27 \mathrm{C} \\
\pm 1.20\end{array}$ & $\begin{array}{c}162.59 \mathrm{D} \\
\pm 1.85\end{array}$ & & $\begin{array}{c}82.60 \mathrm{C} \\
\pm 1.41\end{array}$ & \pm 0.64 \\
\hline & & $\begin{array}{c}83.60 \mathrm{D} \\
\pm 0.99\end{array}$ & $\begin{array}{c}141.72 \mathrm{D} \\
\pm 1.22\end{array}$ & $\begin{array}{c}32.58 \mathrm{D} \\
\pm 0.63\end{array}$ & $\begin{array}{c}96.63 \mathrm{E} \\
\pm 0.81\end{array}$ & $\begin{array}{c}188.17 \mathrm{C} \\
\pm 0.68\end{array}$ & $\begin{array}{c}56.60 \mathrm{C} \\
\pm 2.41\end{array}$ & $\begin{array}{c}121.40 \mathrm{C} \\
\pm 0.95\end{array}$ & $\begin{array}{c}94.34 \mathrm{D} \\
\pm 0.86\end{array}$ \\
\hline $\begin{array}{l}\text { L.S.D. } \\
\end{array}$ & 3.2308 & 3.8368 & 5.3162 & 3.0040 & 5.0495 & 17.614 & 5.0834 & 5.6831 & 4.5247 \\
\hline
\end{tabular}

Data presented as the means of three replicates \pm SD. Different letters refer to significant difference $(\mathrm{P} \leq 0.05)$. 
Table 5. Effect of AMF, T. harzianum and AMF + T. harzianum biological treatments on leaves content of Phenols, Amino acids and Reducing sugars after 8 weeks in three varieties of tomato infected with Fusarium oxysporum f. sp. lycopersici.

\begin{tabular}{|c|c|c|c|c|c|c|c|c|c|}
\hline \multirow{2}{*}{ Variety } & \multicolumn{3}{|c|}{ Castle-rock } & \multicolumn{3}{c|}{ Super Marmande } & \multicolumn{3}{c|}{ Peto 86 } \\
\cline { 2 - 10 } Treatment & Phenols & $\begin{array}{c}\text { Amino } \\
\text { acids }\end{array}$ & $\begin{array}{c}\text { Reducing } \\
\text { sugars }\end{array}$ & Phenols & $\begin{array}{c}\text { Amino } \\
\text { acids }\end{array}$ & $\begin{array}{c}\text { Reducing } \\
\text { sugars }\end{array}$ & Phenols & $\begin{array}{c}\text { Amino } \\
\text { acids }\end{array}$ & $\begin{array}{c}\text { Reducing } \\
\text { sugars }\end{array}$ \\
\hline AMF & $\begin{array}{c}92.50 \mathrm{C} \\
\pm 2.94\end{array}$ & $\begin{array}{c}145.35 \mathrm{C} \\
\pm 0.99\end{array}$ & $\begin{array}{c}73.06 \mathrm{C} \\
\pm 2.33\end{array}$ & $\begin{array}{c}103.58 \mathrm{D} \\
\pm 1.06\end{array}$ & $\begin{array}{c}175.66 \mathrm{~B} \\
\pm 1.65\end{array}$ & $\begin{array}{c}35.23 \mathrm{C} \\
\pm 1.97\end{array}$ & $\begin{array}{c}106.30 \mathrm{C} \\
\pm 0.75\end{array}$ & $\begin{array}{c}103.09 \mathrm{C} \\
\pm 0.73\end{array}$ & $\begin{array}{c}16.73 \mathrm{E} \\
\pm 1.34\end{array}$ \\
\hline $\begin{array}{c}\text { T. har- } \\
\text { zianum }\end{array}$ & $\begin{array}{c}75.08 \mathrm{D} \\
\pm 3.14\end{array}$ & $\begin{array}{c}166.97 \mathrm{~A} \\
\pm 0.99\end{array}$ & $\begin{array}{c}98.52 \mathrm{~A} \\
\pm 2.30\end{array}$ & $\begin{array}{c}112.51 \mathrm{C} \\
\pm 0.90\end{array}$ & $\begin{array}{c}152.24 \mathrm{C} \\
\pm 0.83\end{array}$ & $\begin{array}{c}35.44 \mathrm{C} \\
\pm 0.51\end{array}$ & $\begin{array}{c}126.94 \mathrm{~B} \\
\pm 1.26\end{array}$ & $\begin{array}{c}152.38 \mathrm{~B} \\
\pm 0.64\end{array}$ & $\begin{array}{c}54.95 \mathrm{~B} \\
\pm 1.16\end{array}$ \\
\hline $\begin{array}{c}\text { AMF }+\boldsymbol{T} \text {. } \\
\text { harzianum }\end{array}$ & $\begin{array}{c}187.05 \mathrm{~A} \\
\pm 1.41\end{array}$ & $\begin{array}{c}150.56 \mathrm{~B} \\
\pm 1.26\end{array}$ & $\begin{array}{c}84.65 \mathrm{~B} \\
\pm 0.30\end{array}$ & $\begin{array}{c}184.64 \mathrm{~A} \\
\pm 2.01\end{array}$ & $\begin{array}{c}192.47 \mathrm{~A} \\
\pm 1.73\end{array}$ & $\begin{array}{c}64.57 \mathrm{~A} \\
\pm 1.64\end{array}$ & $\begin{array}{c}157.25 \mathrm{~A} \\
\pm 0.90\end{array}$ & $\begin{array}{c}162.64 \mathrm{~A} \\
\pm 1.38\end{array}$ & $\begin{array}{c}63.53 \mathrm{~A} \\
\pm 1.00\end{array}$ \\
\hline $\begin{array}{c}\text { Control } \\
\text { (autoclaved } \\
\text { soil) }\end{array}$ & $\begin{array}{c}129.69 \mathrm{~B} \\
\pm 0.67\end{array}$ & $\begin{array}{c}92.14 \mathrm{D} \\
\pm 1.53\end{array}$ & $\begin{array}{c}75.52 \mathrm{C} \\
\pm 2.02\end{array}$ & $\begin{array}{c}73.11 \mathrm{E} \\
\pm 2.68\end{array}$ & $\begin{array}{c}141.34 \mathrm{D} \\
\pm 0.63\end{array}$ & $\begin{array}{c}56.90 \mathrm{~B} \\
\pm 1.64\end{array}$ & $\begin{array}{c}93.67 \mathrm{D} \\
\pm 0.53\end{array}$ & $\begin{array}{c}94.32 \mathrm{D} \\
\pm 0.71\end{array}$ & $\begin{array}{c}42.40 \mathrm{C} \\
\pm 1.77\end{array}$ \\
\hline $\begin{array}{c}\text { Control } \\
\text { (infested }\end{array}$ & $82.42 \mathrm{DC}$ \\
soil) & 59.67 & $\begin{array}{c}52.56 \mathrm{E} \\
\pm 2.07\end{array}$ & $\begin{array}{c}10.43 \mathrm{D} \\
\pm 0.47\end{array}$ & $\begin{array}{c}122.68 \mathrm{~B} \\
\pm 2.20\end{array}$ & $\begin{array}{c}156.27 \mathrm{C} \\
\pm 1.71\end{array}$ & $\begin{array}{c}52.23 \mathrm{~B} \\
\pm 2.31\end{array}$ & $\begin{array}{c}155.40 \mathrm{~A} \\
\pm 3.12\end{array}$ & $\begin{array}{c}91.90 \mathrm{D} \\
\pm 2.46\end{array}$ & $\begin{array}{c}37.43 \mathrm{D} \\
\pm 0.91\end{array}$ \\
\hline L.S.D. & 15.746 & 4.6963 & 5.7296 & 6.2529 & 4.5909 & 5.6705 & 5.3065 & 4.5215 & 4.1905 \\
\hline
\end{tabular}

Data presented as the means of three replicates \pm SD. Different letters refer to significant difference $(\mathrm{P} \leq$ $0.05)$.

Amino acids contents were higher in tomato leaves treated by AMF, T. harzianum and AMF $+T$. harzianum treatments in comparison with infected and healthy control (Table 5).

Reducing sugars contents were higher in tomato leaves treated with $\mathrm{AMF}+T$. harzianum and T. harzianum in castle-rock and peto 86 in comparision with infected and healthy control (Table 5). While reducing sugars in super marmande variety had the highest value (64.57 $\mathrm{mg} / 100 \mathrm{~g}$ f.wt.) in $\mathrm{AMF}+T$. harzianum treatment, but was lower in AMF and $T$. harzianum treatments( 35.23 and $35.44 \mathrm{mg} / 100 \mathrm{~g}$.f.wt.) in comparison with infected and healthy control (52.23and $56.90 \mathrm{mg} / 100 \mathrm{~g}$ .f.wt )

In general phenolic acids, amino acids and reducing sugars contents showed higher increased in roots and leaves of tomato vareties in most treatments AMF, T. harzianum and $\mathrm{AMF}+T$. harzianum, This might be due to play an important role in resistance and defense against $F$. oxysporum. $\mathrm{AMF}+T$. harzianum treatment gave the highest contents of phenolic acids, amino acids and reducing sugars

The phenolic content was recorded to be higher in all the treatments viz. $F$. oxysporum, $F$. oxysporum $+($ Trichoderma harzianum)TH , F. oxysporum + (salicylic acid) SA and $F$. oxysporum + $\mathrm{TH}+\mathrm{SA}$ than that of the healthy plants and reached its maximum level in the plants treated with $F$. oxysporum $+\mathrm{TH}+\mathrm{SA} . F$. oxysporum infection resulted in the induction of both of the peroxidase and polyphenol oxidase enzyme activities but at a lower level The combined application of SA (1.5 mM) and TH in Fusarium infected tomato plants, also enhanced the activities of both of the enzymes compared to the non infected or infected(Ojha and Chatterjee, 2012). 
Benhamou et al., (1994) have suggested that phenols and Chitinase were involved in plant resistance to fungal pathogen induced by AM fungi. Fry (1987) demonstrated that the cell wall bound phenols are important because they are the sites at which the covalent cross links may form by oxidative coupling between wall polymers.

The AM ( Glomus fasciculatum) fungus-infected tomato plants showed an increase in reducing and total sugars both in leaves and roots, but more in leaves (Raman and Gnanaguru, 2015). Leaves of AM fungus-infected plants generally contain more sucrose, reducing sugars and starch than non-mycorrhizal plants (Dixon et al., 1988; Nemec and $\mathrm{Vu}, 1990)$. The increased level of reducing sugars in mycorrhizal roots lowers disease incidence (Schenck, 1981)

Generally mycorrhizal association increases the amino acid content in plants. An increased level of amino acids was found in AM (Glomus fasciculatum) fungus-infected tomato plants (Raman and Gnanaguru, 2015). Krishna and Bagyaraj (1983) found higher level of amino acids in Arachis hypogea inoculated with $G$. fasciculatum. Increase in amino acid level was directly correlated with increase in AM fungal infection (Dehne, 1986). Such increase in free amino acids of AM infected plants was observed in G. fasciculatum inoculated tomato plants. The amino acid content decreased in leaves and roots of infected control (Ratnayake et al., 1978).

Data in Tables (6) and (7) show the significant differences between treatments in phenylalanine ammonia lyase, ascorbate peroxidase, peroxidase and polyphenoloxidase enzymes after 8 weeks in three varieties of tomato roots and leaves respectively infected with Fusarium oxysporum f. sp. lycopersici.

AMF, T. harzianum and AMF + T. harzianum gave the highest POD activity in tomato roots of all tested varieties in comparision of infected and healthy control (Table 6). while peto 86 variety had the highest POD activity in tomato roots treated with $\mathrm{AMF}+T$. harzianum and $T$. harzianum (35550 and 31010.33 unit $/ \mathrm{mg}$ protein), while AMF treatment gave the lowest activity( 14308.33 unit /mg protein) in comparison with infected and healthy control(17181.67 and 8516 unit /mg protein) respectively. PPO activity was higher in tomato roots treated with $\mathrm{AMF}+T$. harzianum and AMF in all varieties in comparison with infected and non infected control.

Apx activity in tomato roots of castle-rock variety was higher in $\mathrm{AMF}+T$. harzianum, T. harzianum and AMF, treatments $(90,84.67$ and 34.67 unit $/ \mathrm{mg}$ protein), in comparison to infected and non infected control (25.331 and 22.33 unit /mg protein). While super marmande tomato leaves had a higher apx activity by $\mathrm{AMF}+T$. harzianum, and AMF treatments. but activity was higher peto 86 variety treated with AMF + $T$. harzianum and T. harzianum in comparison to infected and non infected control( Table 6 ) .

PAL activity was higher in tomato roots of castle-rock and super marmande treated with $\mathrm{AMF}+T$. harzianum and T. harzianum, while activity was higher in tomato roots of 
peto 86 in all treatment in comparison to infected and non infected control (Table 6).

POD activity in tomato leaves of super marmande and peto 86 varieties was higher in all treatments in comparison with infected control( Table 7), while almost higher in castle-rock treated with $\mathrm{AMF}+T$. harzianum, and AMF (16531 and 10531.33 unit $/ \mathrm{mg}$ protein) (Table 7) in comparison with infected or healthy control ( 8021 or 3026.67 unit $/ \mathrm{mg}$ protein ). Castle-rock and peto 86 varieties leaves had a higher PPO activity in $\mathrm{AMF}+T$. harzianum, and AMF treatments. While super marmande had a higher PPO activity in $\mathrm{AMF}+T$. harzianum and T. harzianum treatments (3851.33 and 2750.33 unit /mg protein) in comparison with infected and non infected control. (1879.67 and 2361.33 unit /mg protein). 
Table 6. Effect of AMF, T. harzianum and AMF + T. harzianum biological treatments on the activity of phenylalanine ammonia lyase (unit/ mg protein), ascorbate peroxidase (unit / mg protein), peroxidase (unit / mg protein) and poly phenoloxidase (unit / mg protein) after 8 weeks in three varieties of tomato roots infected with Fusarium oxysporum f. sp. lycopersici.

\begin{tabular}{|c|c|c|c|c|c|c|c|c|c|c|c|c|}
\hline \multirow{2}{*}{$\begin{array}{l}\text { Variety } \\
\text { Treatment }\end{array}$} & \multicolumn{4}{|c|}{ Castle-rock } & \multicolumn{4}{|c|}{ Super Marmande } & \multicolumn{4}{|c|}{ Peto 86} \\
\hline & PAL R & $\operatorname{apx}$ & POD & PPO & PAL & $\operatorname{apx}$ & POD & PPO & PAL & $\operatorname{apx}$ & POD & PPO \\
\hline AMF & $\begin{array}{c}2361.33 \mathrm{C} \\
\pm 6.55\end{array}$ & $\begin{array}{c}34.67 \mathrm{C} \\
\pm 0.47\end{array}$ & $\begin{array}{c}14441.67 \mathrm{C} \\
\pm 8.18\end{array}$ & $\begin{array}{c}7020.33 \mathrm{~B} \\
\pm 2.87\end{array}$ & $\begin{array}{c}1149.67 \mathrm{E} \\
\pm 5.73\end{array}$ & $\begin{array}{c}44.67 \mathrm{~B} \\
\pm 0.47\end{array}$ & $\begin{array}{c}40080.33 \mathrm{~B} \\
\pm 5.31\end{array}$ & $\begin{array}{c}13708.67 \mathrm{~B} \\
\pm 4.50\end{array}$ & $\begin{array}{c}5309.00 \mathrm{~B} \\
\pm 2.94\end{array}$ & $\begin{array}{c}17.33 \mathrm{E} \\
\pm 0.47\end{array}$ & $\begin{array}{c}14308.33 \mathrm{D} \\
\pm 6.24\end{array}$ & $\begin{array}{c}8991.00 \mathrm{~B} \\
\pm 4.90\end{array}$ \\
\hline $\begin{array}{l}\text { T. har- } \\
\text { zianum }\end{array}$ & $\begin{array}{c}3891.33 \mathrm{~B} \\
\pm 4.19\end{array}$ & $\begin{array}{c}84.67 \mathrm{~B} \\
\pm 0.47\end{array}$ & $\begin{array}{c}43706.33 \mathrm{~A} \\
\pm 3.68\end{array}$ & $\begin{array}{c}4127.00 \mathrm{D} \\
\pm 4.55\end{array}$ & $\begin{array}{c}2952.00 \mathrm{~A} \\
\pm 3.56\end{array}$ & $\begin{array}{c}16.33 \mathrm{E} \\
\pm 0.47\end{array}$ & $\begin{array}{c}22420.67 \mathrm{C} \\
\quad \pm 6.55\end{array}$ & $\begin{array}{c}6419.00 \mathrm{C} \\
\pm 4.90\end{array}$ & $\begin{array}{c}2657.00 \mathrm{C} \\
\quad \pm 4.32\end{array}$ & $\begin{array}{c}54.33 \mathrm{~B} \\
\pm 0.47\end{array}$ & $\begin{array}{c}31010.33 \mathrm{~B} \\
\pm 3.69\end{array}$ & $\begin{array}{c}4121.67 \mathrm{D} \\
\pm 5.44\end{array}$ \\
\hline $\begin{array}{l}\mathrm{AMF}+T . \\
\text { harzianum }\end{array}$ & $\begin{array}{c}4448.67 \mathrm{~A} \\
\pm 154.76\end{array}$ & $\begin{array}{c}90.00 \mathrm{~A} \\
\pm 1.63\end{array}$ & $\begin{array}{c}18465.00 \mathrm{~B} \\
\pm 40.47\end{array}$ & $\begin{array}{c}8229.33 \mathrm{~A} \\
\pm 4.78\end{array}$ & $\begin{array}{c}2590.67 \mathrm{~B} \\
\pm 7.36\end{array}$ & $\begin{array}{c}58.00 \mathrm{~A} \\
\pm 1.63\end{array}$ & $\begin{array}{c}45187.00 \mathrm{~A} \\
\pm 441.03\end{array}$ & $\begin{array}{c}14313.00 \mathrm{~A} \\
\pm 58.03\end{array}$ & $\begin{array}{c}5987.33 \mathrm{~A} \\
\quad \pm 1.2\end{array}$ & $\begin{array}{c}85.00 \mathrm{~A} \\
\pm 1.63\end{array}$ & $\begin{array}{c}35550.00 \mathrm{~A} \\
\quad \pm 1.64\end{array}$ & $\begin{array}{c}9965.33 \mathrm{~A} \\
\pm 2.05\end{array}$ \\
\hline $\begin{array}{c}\text { Control } \\
\text { (autoclaved } \\
\text { soil) }\end{array}$ & $\begin{array}{c}2327.67 \mathrm{C} \\
\pm 12.50\end{array}$ & $\begin{array}{c}22.33 \mathrm{E} \\
\pm 0.47\end{array}$ & $\begin{array}{c}13232.33 \mathrm{D} \\
\pm 4.19\end{array}$ & $\begin{array}{c}6950.00 \mathrm{C} \\
\pm 2.45\end{array}$ & $\begin{array}{c}2528.00 \mathrm{C} \\
\pm 4.55\end{array}$ & $\begin{array}{c}36.33 \mathrm{C} \\
\pm 0.41\end{array}$ & $\begin{array}{c}14567.33 \mathrm{D} \\
\pm 5.44\end{array}$ & $\begin{array}{c}4160.00 \mathrm{E} \\
\quad \pm 5.72\end{array}$ & $\begin{array}{c}1911.00 \mathrm{E} \\
\pm 3.74\end{array}$ & $\begin{array}{c}27.67 \mathrm{D} \\
\pm 0.47\end{array}$ & $\begin{array}{c}8516.00 \mathrm{E} \\
\pm 4.09\end{array}$ & $\begin{array}{c}2121.00 \mathrm{E} \\
\pm 3.75\end{array}$ \\
\hline $\begin{array}{c}\text { Control } \\
\text { (infested } \\
\text { soil) }\end{array}$ & $\begin{array}{c}2450.67 \mathrm{C} \\
\pm 3.30\end{array}$ & $\begin{array}{c}25.33 \mathrm{D} \\
\pm 0.47\end{array}$ & $\begin{array}{c}8788.67 \mathrm{E} \\
\pm 3.68\end{array}$ & $\begin{array}{c}3539.67 \mathrm{E} \\
\pm 4.92\end{array}$ & $\begin{array}{c}1366.67 \mathrm{D} \\
\pm 5.31\end{array}$ & $\begin{array}{c}29.67 \mathrm{D} \\
\pm 0.47\end{array}$ & $\begin{array}{c}14219.00 \mathrm{D} \\
\pm 3.27\end{array}$ & $\begin{array}{c}4821.00 \mathrm{D} \\
\quad \pm 4.90\end{array}$ & $\begin{array}{c}2356.67 \mathrm{D} \\
\pm 6.24\end{array}$ & $\begin{array}{c}49.33 \mathrm{C} \\
\pm 0.47\end{array}$ & $\begin{array}{c}17181.67 \mathrm{C} \\
\pm 4.11\end{array}$ & $\begin{array}{c}4868.00 \mathrm{C} \\
\pm 3.75\end{array}$ \\
\hline L.S.D. & 228.86 & 2.7753 & 61.563 & 13.328 & 17.946 & 2.7753 & 649.31 & 86.686 & 13.31 & 2.7753 & 13.859 & 13.631 \\
\hline
\end{tabular}

Data presented as the means of three replicates \pm SD. Different letters refer to significant difference $(\mathrm{P} \leq 0.05)$.

Ascorbate peroxidase (APX) activity had the highest value in all leaves of tested varieties treated with $\mathrm{AMF}+T$. harzianum in comparison with other treatments. PAL activity was higher in castle-rock variety leaves treated with $\mathrm{AMF}+T$. harzianum (10762.67 unit /mg protein) , while activity was higher in super marmande and peto 86 varieties treated with $\mathrm{AMF}+T$. harzianum and AMF.

In general POD, PPO, APX and PAL activities showed higher increased in roots and leaves of tomato vareties in most treatments AMF, $T$. harzianum and $\mathrm{AMF}+T$. harzianum ,This might be due to play an important role in resistance and defense against $F$. oxysporum. AMF $+T$. harzianum treatment gave the highest activity of POD, PPO, APX and PAL.

Earlier researchers observed enhanced activity of peroxidase and polyphenol oxidase enzyme activities in host tissues in response to pathogenic infection (Ojha et al. 2005 and Chakraborty and Chatterjee 2007). Toxic metabolites of the fungus may activate the production of phenoloxidizing enzymes. Fusarium species are known to produce such metabolites which also play a vital role in the tissue browning by their ability to oxidize phenols to quinines which are known to be more reactive and have more antimicrobial activity than the phenols, already exist in plants infected with $F$. oxysporum and $\mathrm{TH}$ treated (Morkunas and Gmerek, 2007). On the other hand, development of an antioxidant defense system in plants protect them against oxidative stress damage by either partial suppression of reactive oxygen species production or the scavenging of reactive oxygen species which are generated during plant pathogen in- 
teractions (Ye et al. 2006; Cavalcanti et al. 2007) Thus, various antioxidant enzymes like peroxidases and polyphenol oxidases can participate in reactive oxygen metabolism of the species during infection.

Significant increases in peroxidase (PO and phenyl alanine ammonialayase (PAL) have been observed associated with bio-and chemical agents treatment in the treated plants which indicated to induction of systemic resistance against $F$. oxysporum. Several studies reported that systemic resistance is characterized by induction of several formation of PRs and has been taken as a marker of the induced resistance
(Kessman et al. 1994). Some of these PRs are PO and PAL enzymes play a role in phenolic compound metabolisms. Other studies indicated to the activation of large amount of enzymes including peroxidase, chitinase, $\beta-1,3$-glucanase, and phenyl alanine ammonia-layase upon treatment of plants with different agents biotic and abiotic. These activation were found associated with cellular alteration in the epidermal and cortical cells that inhibited further colonization and inhibited the causal pathogen to reach the vascular tissue (Hoffland et al. 1995).

Table 7. Effect of AMF, T. harzianum and AMF + T. harzianum biological treatments on the activity of phenylalanine ammonia lyase (unit/ $\mathrm{mg}$ protein), ascorbate peroxidase (unit / mg protein), peroxidase (unit / $\mathrm{mg}$ protein) and poly phenoloxidase (unit / $\mathrm{mg}$ protein) after 8 weeks in three varieties of tomato leaves infected with Fusarium oxysporum f. sp. lycopersici.

\begin{tabular}{|c|c|c|c|c|c|c|c|c|c|c|c|c|}
\hline \multirow{2}{*}{$\begin{array}{c}\text { Variety } \\
\text { Treatment }\end{array}$} & \multicolumn{4}{|c|}{ Castle-rock } & \multicolumn{4}{|c|}{ Super Marmande } & \multicolumn{4}{|c|}{ Peto 86} \\
\hline & PAL & $\operatorname{apx}$ & POD & PPO & PAL & apx & POD & PPO & PAL & apx & POD & PPO \\
\hline AMF & $\begin{array}{c}7678.00 \mathrm{C} \\
\pm 2.94\end{array}$ & $\begin{array}{c}10.33 \mathrm{C} \\
\pm 0.47\end{array}$ & $\begin{array}{c}10531.33 \mathrm{~B} \\
\pm 3.40\end{array}$ & $\begin{array}{c}4058.33 \mathrm{~B} \\
\pm 3.30\end{array}$ & $\begin{array}{c}11039.00 \mathrm{~B} \\
\pm 2.45\end{array}$ & $\begin{array}{l}4.67 \mathrm{D} \\
\pm 0.47\end{array}$ & $\begin{array}{c}2687.33 \mathrm{C} \\
\pm 3.86\end{array}$ & $\begin{array}{c}1808.67 \mathrm{C} \\
\pm 4.99\end{array}$ & $\begin{array}{c}13748.00 \mathrm{~B} \\
\pm 7.48\end{array}$ & $\begin{array}{l}9.67 \mathrm{D} \\
\pm 0.47\end{array}$ & $\begin{array}{c}16986.33 \mathrm{~B} \\
\pm 5.79\end{array}$ & $\begin{array}{c}7980.67 \mathrm{~B} \\
\pm 5.31\end{array}$ \\
\hline $\begin{array}{l}\text { T. har- } \\
\text { zianum }\end{array}$ & $\begin{array}{c}2378.33 \mathrm{E} \\
\quad \pm 4.92\end{array}$ & $\begin{array}{l}7.33 \mathrm{D} \\
\pm 0.47\end{array}$ & $\begin{array}{c}2229.33 \mathrm{E} \\
\quad \pm 5.31\end{array}$ & $\begin{array}{c}690.33 \mathrm{E} \\
\pm 2.87\end{array}$ & $\begin{array}{c}6868.67 \mathrm{D} \\
\pm 4.50\end{array}$ & $\begin{array}{l}1.67 \mathrm{E} \\
\pm 0.47\end{array}$ & $\begin{array}{c}3446.67 \mathrm{~B} \\
\pm 472.15\end{array}$ & $\begin{array}{c}2750.33 \mathrm{~B} \\
\pm 4.50\end{array}$ & $\begin{array}{c}5426.67 \mathrm{E} \\
\quad \pm 4.50\end{array}$ & $\begin{array}{c}21.67 \mathrm{~B} \\
\pm 0.47\end{array}$ & $\begin{array}{c}5226.67 \mathrm{D} \\
\pm 4.78\end{array}$ & $\begin{array}{c}1721.67 \mathrm{E} \\
\pm 3.09\end{array}$ \\
\hline $\begin{array}{c}\mathrm{AMF}+T \\
\text { harzianum }\end{array}$ & $\begin{array}{c}10762.67 \mathrm{~A} \\
\pm 30.18\end{array}$ & $\begin{array}{c}29.33 \mathrm{~A} \\
\pm 1.25\end{array}$ & $\begin{array}{c}16531.00 \mathrm{~A} \\
\pm 197.32\end{array}$ & $\begin{array}{c}6329.67 \mathrm{~A} \\
\pm 52.82\end{array}$ & $\begin{array}{c}12050.00 \mathrm{~A} \\
\pm 43.204\end{array}$ & $\begin{array}{c}23.67 \mathrm{~A} \\
\pm 1.70\end{array}$ & $\begin{array}{c}6316.00 \mathrm{~A} \\
\pm 145.84\end{array}$ & $\begin{array}{c}3851.33 \mathrm{~A} \\
\pm 490.04\end{array}$ & $\begin{array}{c}14709.00 \mathrm{~A} \\
\pm 63.04\end{array}$ & $\begin{array}{c}28.67 \mathrm{~A} \\
\pm 0.94\end{array}$ & $\begin{array}{c}17871.33 \mathrm{~A} \\
\pm 4.64\end{array}$ & $\begin{array}{c}8722.67 \mathrm{~A} \\
\pm 95.00\end{array}$ \\
\hline $\begin{array}{c}\text { Control } \\
\text { (autoclaved } \\
\text { soil) } \\
\end{array}$ & $\begin{array}{c}8477.00 \mathrm{~B} \\
\quad \pm 2.94\end{array}$ & $\begin{array}{l}5.33 \mathrm{D} \\
\pm 0.47\end{array}$ & $\begin{array}{c}3026.67 \mathrm{D} \\
\pm 4.99\end{array}$ & $\begin{array}{c}2150.67 \mathrm{D} \\
\pm 6.55\end{array}$ & $\begin{array}{c}4007.00 \mathrm{E} \\
\quad \pm 3.56\end{array}$ & $\begin{array}{c}11.67 \mathrm{C} \\
\pm 0.47\end{array}$ & $\begin{array}{c}5937.33 \mathrm{~A} \\
\pm 4.64\end{array}$ & $\begin{array}{c}2361.33 \mathrm{CB} \\
\pm 3.40\end{array}$ & $\begin{array}{c}9960.33 \mathrm{D} \\
\pm 2.87\end{array}$ & $\begin{array}{l}6.67 \mathrm{E} \\
\pm 0.47\end{array}$ & $\begin{array}{c}5950.00 \mathrm{C} \\
\pm 5.72\end{array}$ & $\begin{array}{c}4229.33 \mathrm{C} \\
\pm 3.68\end{array}$ \\
\hline $\begin{array}{c}\text { Control } \\
\text { (infested } \\
\text { soil) }\end{array}$ & $\begin{array}{c}5737.33 \mathrm{D} \\
\pm 471.88\end{array}$ & $\begin{array}{c}14.67 \mathrm{~B} \\
\pm 0.47\end{array}$ & $\begin{array}{c}8021.00 \mathrm{C} \\
\pm 2.94\end{array}$ & $\begin{array}{c}3229.33 \mathrm{C} \\
\pm 6.24\end{array}$ & $\begin{array}{c}7529.67 \mathrm{C} \\
\pm 4.11\end{array}$ & $\begin{array}{c}18.67 \mathrm{~B} \\
\pm 0.47\end{array}$ & $\begin{array}{c}1678.67 \mathrm{D} \\
\pm 2.05\end{array}$ & $\begin{array}{c}1879.67 \mathrm{C} \\
\pm 4.92\end{array}$ & $\begin{array}{c}10553.33 \mathrm{C} \\
\pm 4.19\end{array}$ & $\begin{array}{c}18.33 \mathrm{C} \\
\pm 0.47\end{array}$ & $\begin{array}{c}1949.33 \mathrm{E} \\
\pm 2.87\end{array}$ & $\begin{array}{c}2020.00 \mathrm{D} \\
\pm 4.55\end{array}$ \\
\hline L.S.D. & 696.02 & 2.3011 & 290.69 & 79.138 & 64.533 & 2.8607 & 727.38 & 721.37 & 93.966 & 1.9624 & 16.048 & 140.37 \\
\hline
\end{tabular}

Data presented as the means of three replicates \pm SD. Different letters refer to significant difference $(\mathrm{P} \leq 0.05)$. 
Trichoderma viride (Pers.) preinoculated wheat seedlings infected with Fusarium oxysporum Schlecht. mediated activation of antioxidant enzymes such as, catalase, guaiacol peroxidase, ascorbate peroxidase, superoxide dismutase in co-stressed seedlings indicated their involvement in enhanced resistance against Fusarium infection, which is suggestive of playing crucial role in mitigating cellular toxicity developed due to excess $\mathrm{H}_{2} \mathrm{O}_{2}$. Thus, Trichoderma pre-inoculation protected wheat against Fusarium infection by stabilising oxidative stress (Mohapatra and Mittra, 2017).

\section{Conclusion}

AMF, T. harzianum and AMF $+T$. harzianum, treatments induced resistance in tomato varieties against $F$. oxysporum. $\mathrm{AMF}+T$. harzianum treatment was the best treatment.

\section{References}

Agrios, G.N. (1988). Plant Pathology. Third Ed. Academic Press, New

Altomare, C.; Norvell, W.A.; Bjorkman, T.; Harman, G.E. (1999). Solubilization of phosphates and micronutrients by plant growth promoting and biocontrol fungus Trichoderma harzianum strain 1295-22. Appl. Environ. Microbiol. 65, 2926-2933.

Amini, J. (2009). Physiological race of Fusarium oxysporum f. sp. lycopersici in Kurdistan province of Iran and reaction of some tomato cultivars to race 1 of pathogen. Plant Pathol. J. 8, 68-73.

Baker, R. (1989). Improved Trichoderma spp. for promoting crop productivity. Trends Biotechnol. 7, 34-38.

Beaudoin-Eagan, L.D. and T.A.Thorp. (1985). Tyrosine and phenylalanine ammonia lyase activities during shoot initiation in tobacco callus cultures. Plant Physiol., 78 (3):438-441.

Benhamou N, Fortin JA, Hamel C, StArnand M, Shatilla A. (1994). Resistance responses of mycorrhizal $\mathrm{Ri}$ T-DNA-transformed carrot roots to infection by Fusarium oxysporum f. sp. chrysanthemi. Phytopathol. 84, 958-968.

Benjamin, N. and M.W. Montgomery (1973). Polyphenol oxidase of royal ann cherries: purification and characterization. J. Food Sci. 38: 799-806.

Bradford, M.M. (1976). A rapid and sensitive method for the quantitation of microgram quantities of protein utilizing the principal of protein Dye Binding. Anal. Biochem., 72: 248-254

Caron, M. (1989). Potential use of mycorrhizae in soil borne diseases. C. J. Plant Pathol. 11, 177-179.

Cavalcanti F.R., Resende M.L., Lima S.P., Silveira J.A., Oliveira, J.T. (2007). Activities of antioxidant enzymes and photosynthetic responses in tomato pre-treated by plant activators and inoculated by Xanthomonas vesicatoria. Physiol. Mol. Plant Pathol. 68 (4-6): 198208.

Chakraborty M.R., Chatterjee N.C. (2007). Interaction of Trichoderma harzianum with Furarium solani during its pathogenesis and the associated resistance of the host. Asian J. Exp. Sci. 21(2)353-357 .

Chet, I. (1987). Trichodermaapplication, mode of action, and potential as biocontrol agent of soilborne plant pathogenic fungi. InChet, I. (eds). Innovative approaches to plant disease control. John Wiley and Sons, Inc. New york.p.137-160.

Datnoff, L.E.; Nemec, S.; Pernezny, K. (1995). Biological control of Fusa- 
rium crown and root rot of tomato in Florida using T. harzianum and Glomus intraradices. Biol. Control. 5, 427-431.

Declerck, S.; Risede, J.M.; Rufyikiri, G.; Delvaux, B. (2002). Effect of arbuscular mycorrhizal fungi on severity of root rot of bananas caused by Cylindrocladium spathiphylli. Plant Pathol. 51,109-115.

Dehne HW. (1986). Influence of VA Mycorrhizae on host plant physiology. In: S. Gianninazzi-Pearson, Gianninazzi (eds), Physiology and Genetical Aspects of Mycorrhizae. INRA, Paris, 431-435.

Dixon RK, Garrett HE, Fox GS. (1988). Carbohydrate relationships of Citrus jambhiri inoculated with Glomus fasciculatum. J. Am. Soc. Hort. Sci. 113, 239-242.

Dubsk'y, M.; Sr'amek, M.; Vos'atka, M. (2002). Inoculation of Cyclamen (Cyclamen persicum) and Euphorbia pulcherrina with arbuscular mycorrhizal fungi and Trichoderma harzianum. Rostl. Vyroba. 48, 63-68.

Elad, Y. and I. Chet, (1983). Improved selective medium for isolation of Trichoderma or Fusarium sp. Phytoparasitica, 11: 55-58.

Fry SC. (1987). Intracellular feruloyation of pectic polysaccharides. Planta 171, 205-211.

Hage-Ahmed, K., Krammer, J., and Steinkellner, S. (2013). The intercropping partner affects arbuscular mycorrhizal fungi and Fusarium oxysporium f. sp. lycopersici interactions in tomato. Mycorrhiza 23, 543-550. doi: 10.1007/s00572013-0495-X

Hammer Schmidt, R.; E. M. Nuckles and J. Kuc (1982). Association of enhanced peroxidase activity with induced systemic resistance of cucumber to colletotrichum lagenarium. Physiol. Plant., 20:73-82.
Harman, G.E. (2000). Myths and dogmas of biocontrol. Changes in perceptions derived from research on Trichoderma harzianum T-22. Plant Dis. 84, 377-393.

Harman, G.E., (2006). Overview of mechanisms and uses of Trichoderma spp. Phytopathology, 96: 190-194.

Hoffland, E., Pieterse, C.M.J., Bik, L. and Vanpelt, J.A. (1995). Induced systemic resistance in radish is not associated with accumulation of pathogenesis-related proteins. Physiol. Mol. Plant Pathol. 46:309320.

Jayeraman, J. (1985). Laboratory manual in biochemistry. Wiley Eastern Ltd. New Delhi, India., 107.

Johnson, C.R.; Menge, J.A.; Schwab, S.; Ting, I.P. (1982). Interaction of photoperiod and vesicular arbuscular mycorrhizae on growth and metabolism of sweet orange. New Phytol. 90, 665-670.

Jones, J.B.; Jones, J.P.; Stall, R.E.; Zitter, T.A. (1991). Compendium of Tomato Diseases. American Phytopathological Society St. Paul.

Kessman, H., Staub, T., Ligon, J., Oostendorp, H. and Ryals, J.(1994). Activation of systemic acquired disease resistance in plants'.Eur. J. Plant Pathol. 100:359-369.

Krishna KR, Bagyaraj DJ. (1983). Changes in the free amino nitrogen and protein fractions of ground nut caused by inoculation with VAmycorrhiza. Ann. Bot. 51, 399401.

Miller, G.L. (1959). Use of dinitrosalicylic acid reagent for determination of reducing sugar, Anal. Chem., 31, 426.

Mohapatra, S., and B. Mittra (2017). Alleviation of Fusarium oxysporum induced oxidative stress in wheat by Trichoderma viride .Archives of Phytopathology and Plant Pro- 
tection .Volume 50, 2017. Issue12.

Morkunas I., Gmerek J. (2007). The possible involvement of peroxidase in defense of yellow lupin embryos axes against Fusarium oxysporum. J. Plant Physiol. 164 (6): 497-506.

Mwangi, M. W.; Monda, E.O.; Okoth S. A. and Jefwa J.M (2011). Inoculation of tomato seedlings with Trichodera harizianum and Arbscular Mycorrhizal Fungi and their effect on groth and control of wilt in tomato seedlings. Brazilian Journal of Microbiology 42: 508513.

Nakano,Y. and K. Asada (1981). Hydrogen peroxide is scavenged by ascorbate specific peroxidase in spinach chloroplasts. Plant Cell Physiol. 22(5),867-880.

Nelson, P.E.; Toussoun, T.A.; Marascs, W.F.O.(1983). Fusarium species. An illustrated manual for identification. Pennsylvania State University. University Press, Pennsylvania.

Nemec S, Vu JCV. (1990). Effect of soil phosphorus and Glomus intraradices on growth, non-structural carbohydrates and photosynthetic activity on Citrus aurantium. Plant Soil 128, 257-263.

Nemec, S.; Datnoff, L.E.; Strandberg, J. (1996). Efficacy of biocontrol agents in planting mixes to colonize plants and control root diseases of vegetables and citrus. Crop Prot. 15, 735-742.

Ojha S., Chakraborty M.R., Chatterjee N.C. (2005). Activities of phenol oxidizing enzymes in anthracnose disease of Saracaasoca under pathogenesis. Indian Biol. 37 (2): 9-11.

Ojha, S.,and N. C. Chatterjee (2012) Induction of resistance in tomato plants against Fusarium oxysporum F. sp. Lycopersici medi- ated through salicylic acid and Tricoderma harzianum.Journal of Plant Protection Research. 52, (2):220-225.

Ozbay, N.; Newman, E.S.(2004). Effect of $T$. harzianum strains to colonize tomato roots and improve transplant growth. Pak. J. Biol. Sci. 7, 253-257.

Raman N and Gnanaguru M. (2015). Biological control of Fusarium wilt of tomato by Glomus fasciculatum. In: Current trends in Life Sciences Vol.21 -Recent Developments in biocontrol of plant pathogens. (eds), K. Manibhushan Rao and A. Mahadevan, Today and Tomorrow's Printers and Publishers, New Delhi, 21-25.

Ratnayake M, Leonard RT, Menge JA. (1978). Root exudation in relation to supply of phosphorus and its possible relevance to mycorrhizal formation. New Phytol. 81, 543552.

Rifai, M.A. (1969). A revision of the genus Trichoderma. Mycological Papers. 116:1-56.

Schenck NC. (1981). Can mycorrhiza control root disease? Plant Dis. 65, 230-234.

Shahidi, F. and M. Naczk (1995). Methods of analysis and quantification of phenolic compounds. Food phenolic: sources, chemistry, effects and applications. Technomic Publishind Company, Inc: Lancaster, PA, 287-293.

Smith, S.E.; John, J.B.; Smith, F.A.; Bromley, J.L. (1986). Effect of mycorrhizal infection on plant growth, nitrogen and phosphorous nutrition in glass house grown Allium cepa L. New Phytol. 103, 359-373.

Snedecor, G. M. and W. G. Cochran (1980). Statistical methods, Sixth Edition, lowa State Univ. Press, Amer. lowa, USA. 
Sr'amek, F.; Dubsk'y, M.; Vos'atka,, M. (2000). Effect of and T. harzianum on three species of balcony plants. Rostl. vyroba. 46, 127-131.

Waudo, S.W.; Owino, P. O.; Kuria, M. (1995). Control of Fusarium wilt of tomatoes using soil amendments. East Afr. Agric. For. J. 60, 207-217.

Ye S.F., Zhou H.Y., Sun Y., Zou L.Y., $\mathrm{Yu}$ J.Q. (2006). Cinnamic acid causes oxidative stress in cucum- ber roots and promotes incidence of Fusarium wilt. Environ. Exp. Bot. 56 (3): 255-262.

Yedida, I.; Benhamou, N.; Chet, I. (1999). Induction of defense response in cucumber plants $(\mathrm{Cu}$ cumis sativus L.) by the biocontrol agent Trichoderma harzianum. Appl. Environ. Microbiol. 65, 1061-1070. 


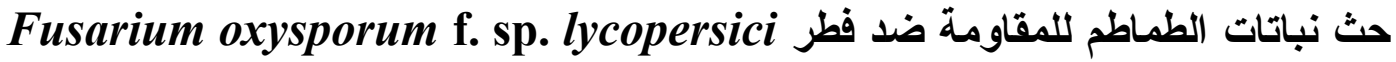
بواسطة فطري الميكور هيز او التريكوديرما.

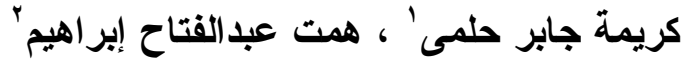

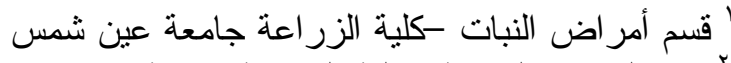

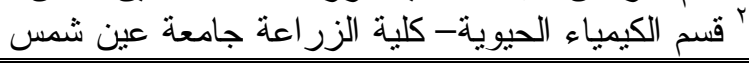

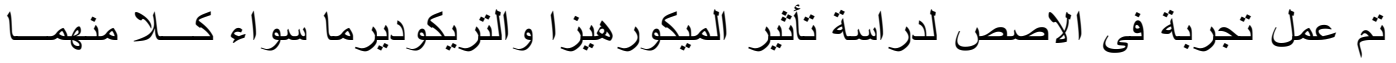

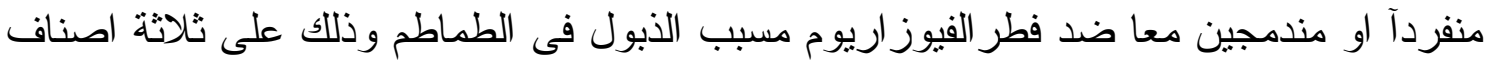

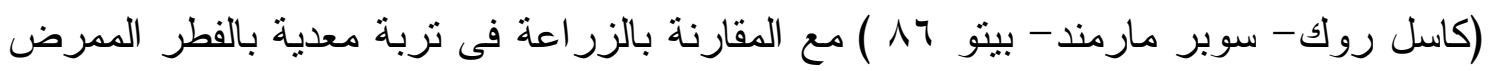

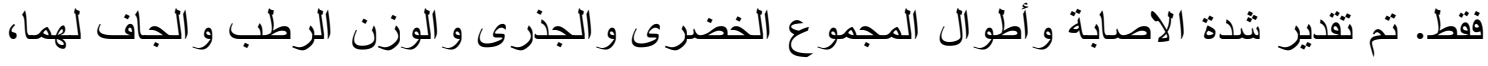

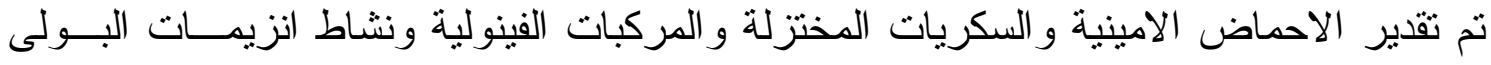

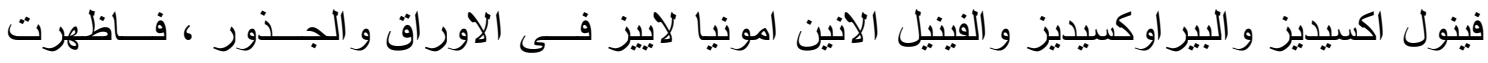

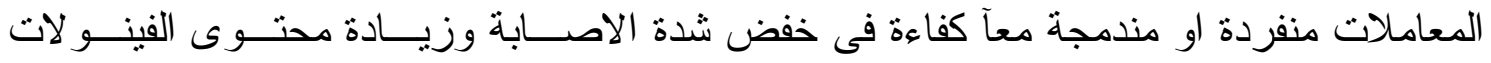

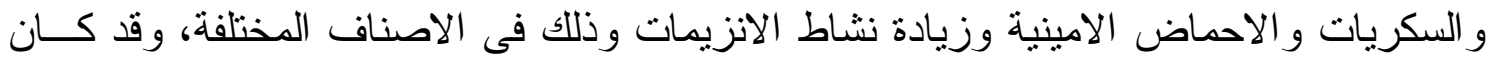

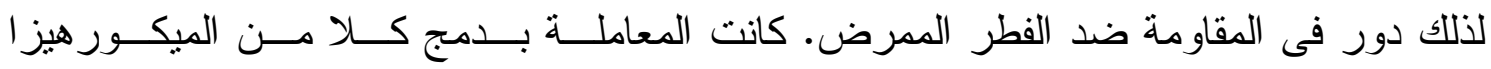
و التزيكوديرما اكثر كفاءة و اعطت افضل نتيجة. 